\title{
On partition function in Astronomy \& Astrophysics
}

\author{
M.K. Sharma ${ }^{1}$, Monika Sharma ${ }^{1}$ and Suresh Chandra ${ }^{2,3}$ \\ ${ }^{1}$ School of Studies in Physics, Jiwaji University, Gwalior 474011 (M.P.), India \\ ${ }^{2}$ Physics Department, Lovely Professional University, Phagwara 144411 (Punjab), India \\ ${ }^{3}$ Zentrum für Astronomie und Astrophysik, Technische Universität Berlin, Hardenbergstrasse \\ 36, D-10623 Berlin, Germany \\ Emails: mohitkumarsharma32@yahoo.in; monika3273@yahoo.in; suresh492000@yahoo.co.in
}

\begin{abstract}
In order to analyze spectrum from the interstellar medium (ISM), spectrum of the molecule of interest is recorded in a laboratory, and accurate rotational and centrifugal distortion constants are derived. By using these constants, one can calculate accurate partition function. However, in the same paper, where these constants are derived, the partition function is calculated by using a semi-empirical expression. We have looked into the details of this semi-empirical expression and compared the values, obtained from it, with the accurate ones. As an example, we have considered the case of Methanimine $\left(\mathrm{CH}_{2} \mathrm{NH}\right)$ which is detected in a number of cosmic objects. It is found that for the kinetic temperature $T>120 \mathrm{~K}$, the semi-empirical expression gives large value as compared to the accurate one. The deviation becomes about $25 \%$ larger than the accurate one at the kinetic temperature of $400 \mathrm{~K}$.
\end{abstract}

Key words. ISM: molecules; Partition function

\section{Introduction}

Partition function derived from the spectroscopic parameters connects to the thermodynamic quantities. From time to time, in various contexts in Astronomy \& Astrophysics, computation of partition function has been a point of extensive discussion. For example, the calculation of partition function of $\mathrm{H}_{3}^{+}$has been in hot discussion in the context of solar continuum observations. Several scientists calculated the partition function of $\mathrm{H}_{3}^{+}$. The partition function of $\mathrm{H}_{3}^{+}$as a function kinetic temperature, calculated by Chandra et al. (1991), was declared by Sidhu et al. (1992) to be the best one. Later on, Neale \& Tennyson (1995) extended the calculation to high temperatures. The rotational partition function $Q$ is expressed as (Chandra et al., 1991; Sidhu et al., 1992; Neale \& Tennyson, 1995)

$$
Q=\sum_{i}\left(2 J_{i}+1\right) \exp \left(-h c E_{i} / k T\right)
$$


Here, $T$ is the kinetic temperature and the nuclear degeneracy is not considered. The $h$, $k, c$ are, respectively, the Planck constant, Boltzmann constant and speed of light. For a rotational level $i$ of asymmetric top molecule, the parameters $J_{i}, E_{i}$ are the rotational quantum number and energy, respectively. The $\left(2 J_{i}+1\right)$ is the rotational statistical weight of level $i$. The values of $J_{i}$ and $E_{i}$ for a level can be calculated by using the rotational and centrifugal distortion constants for the molecule.

However, in many papers where rotational and centrifugal distortion constants are derived with big efforts, the partition function is calculated by using the semi-empirical expression

$$
Q=\sqrt{\frac{\pi}{A B C}\left(\frac{k T}{h}\right)^{3}}
$$

where $A, B$ and $C$ are rotational constants. Details of this expression (2) are discussed in the next section. In the present paper, we have discussed about the deviation obtained when one uses equation (2) in place of (1).

\section{About equation (2)}

Kassel (1933) used partition function for calculation of thermodynamic quantities. Asymptotic expansion of the expression for the rotational partition function of a rigid symmetric top molecule for high temperature $T$ was given by Viney (1933) as the following.

$$
Q=\mathrm{e}^{B h / 4 k T} \sqrt{\frac{\pi}{B^{2} A}\left(\frac{k T}{h}\right)^{3}}\left[1+\frac{1}{12}\left(1-\frac{B}{A}\right) \frac{B h}{k T}+\frac{7}{480}\left(1-\frac{B}{A}\right)^{2}\left(\frac{B h}{k T}\right)^{2}+\ldots\right]
$$

where $A$ and $B$ are rotational constants for the symmetric top molecule.

Since there is no explicit formula for energy of rotational level in case of an asymmetric top molecule, it is impossible to derive a rigorous asymptotic expansion for the partition function in this case. However, Gordon (1934) suggested that for a rigid asymmetric top molecule, in equation (3), $B$ should be replaced by $\sqrt{B C}$ to get the expression as

$$
\begin{aligned}
Q=\mathrm{e}^{\sqrt{B C} h / 4 k T} & \sqrt{\frac{\pi}{A B C}\left(\frac{k T}{h}\right)^{3}}\left[1+\frac{1}{12}\left(1-\frac{\sqrt{B C}}{A}\right) \frac{\sqrt{B C} h}{k T}\right. \\
& \left.+\frac{7}{480}\left(1-\frac{\sqrt{B C}}{A}\right)^{2}\left(\frac{\sqrt{B C} h}{k T}\right)^{2}+\ldots\right]
\end{aligned}
$$

Here, $A, B$ and $C$ are rotational constants for the asymmetric top molecule. This equation (4) may be considered as a semi-empirical expression for a rigid asymmetric top molecule. 
For sufficiently high temperature (or small rotational constants), equation (4) reduces to (2), as the argument of the exponential would be very small, and the contributions of second, third, etc. terms would be neglected. This expression (2) is thus a semiempirical relation derived for a rigid asymmetric top molecule and is valid at sufficiently high temperature. Moreover, the expression (4) is better than (2). But, this expression (2) is being used regularly by the scientists. Probably they are not aware of the facts about the expression (2), and as a tradition, this expression is being used.

\section{Results and discussion}

Now, we have three equations (1), (2) and (4), for the partition function. Let us denote their values as $Q_{1}, Q_{2}, Q_{3}$, respectively, for convenience. There are many papers where accurate rotational and centrifugal distortion constants of asymmetric top molecule are derived, and in the same paper the partition function is calculated with the help of equation (2). Some examples of such papers are: Widicus et al. (2003) (for $\mathrm{NH}_{2} \mathrm{CH}_{2} \mathrm{CH}_{2} \mathrm{OH}$ ), Widicus Weaver et al. (2005) (for $\mathrm{CHOCH}_{2} \mathrm{OH}$ ), Motoki et al. (2014) (for $\mathrm{CH}_{2} \mathrm{NH}$ ). We have not intention about them. We have considered the case of Methanimine $\left(\mathrm{CH}_{2} \mathrm{NH}\right)$ and have used the data of Motoki et al. (2014), given in Table 1.

Using the values given in Table 1, we have calculated accurate energies of rotational levels with the help of the computer code ASROT (Kisiel, 2001). For calculation of partition function, we have considered the levels having energy up to $1475 \mathrm{~cm}^{-1}$, which is sufficiently large for the kinetic temperature up to $400 \mathrm{~K}$, considered here. Using these energies of levels, the partition function is calculated with the help of equation (1). At $400 \mathrm{~K}$, the contribution of the highest level considered is less than $0.01 \%$. It shows that the considered set of energy levels is sufficient, and further high energy levels would not contribute to the partition function significantly, up to a temperature of $400 \mathrm{~K}$.

The results obtained $Q_{1}$ and $Q_{2}$ are presented in Figure 1 as a function of kinetic temperature $T$ in the gas. The values of partition function reported by Motoki et al. (2014) were obtained after multiplying by a factor of 3 for the nuclear degeneracy. Therefore, they differ by a factor of 3 as compared to those obtained with the help of equation (2).

As obvious, the $Q_{3}$ is always larger than $Q_{2}$. For the temperature $T<120 \mathrm{~K}$, the deviation of $Q_{2}$ with respect to $Q_{1}$ is less than $1 \%$, and $Q_{2}$ is always smaller than $Q_{1}$. For $T>120 \mathrm{~K}$, the deviation of $Q_{2}$ with respect to $Q_{1}$ increases with temperature. The deviation becomes $25 \%$ at $T=400 \mathrm{~K}$. It is quite large, and the values of thermodynamic quantities would change significantly. 
The contribution of centrifugal distortion constants to partition function increases when we go to the levels with large value of $J$. In some molecules, the contribution even may change the sequence of levels (Sharma et al., 2014). It is interesting to note that equation (2) does not give accurate results even at high temperatures.

\section{Conclusion}

It may finally be concluded that when the rotational and centrifugal distortion constants are available, the partition function should be calculated with the help of equation (1). It may require some efforts, but the reliable results would be available in literature.

\section{Acknowledgments}

Suresh Chandra thankfully acknowledges nice hospitality of Prof. Dr. D. Breitschwerdt and Prof. Dr. W.H. Kegel of Technical University, Berlin, Germany, where this investigation was finalized. He is grateful to the Alexander von Humboldt Foundation, Bonn (Germany), for financial support and to the Lovely Professional University, Punjab (India) for support and encouragement.

\section{References}

Chandra, S., Gaur, V.P. and Pande, M.C: 1991, J. Quant. Spectrosc. Rad. Trans. 45, 57

Gordon, A.R.: 1934, J. Chem. Phys. 2, 65.

Kassel, L.S.: 1933, J. Chem. Phys. 1, 576.

Z. Kisiel, in: J. Demaison, et al. (Eds.), Spectroscopy from Space, Kluwer, Dordrecht, 2001, pp. 91.

Motoki, Y., Isobe, F., Ozeki, H. and Kobayashi, K.: 2014, Astron. Astrophys. 566, A28.

Neale, L. and Tennyson, J.: 1995, Astrophys. J. 454, L169.

Sharma, M., Sharma, M.K.,Verma, U.P. and Chandra, S.: 2014, Adv. Space Res. 54, 252.

Sidhu, K.S., Miller, S. and Tennyson, J.: 1992, Astron. Astrophys. 255, 453.

Viney, I.E.: 1933, Proc. Cambr. Phil. Soc. 29, 142. 
Widicus, S.L., Drouin, B.J., Dyl, K.A. and Blake, G.A.: J. Mol. Spectr. 217, 278.

Widicus Weaver, S.L., Butler, R.A.H., Drouin, B.J., Petkie, D.T., Dyl, K.A. and De Lucia, F.C.: 2005, Astrophys. J. Suppl. 158, 188. 
Table 1. Rotational and centrifugal distortion constants in $\mathrm{MHz}$.

\begin{tabular}{|c|c|c|c|}
\hline Parameter & Value & Parameter & Value \\
\hline $\bar{A}$ & 196210.87629 & $H_{J K}$ & $2.6068 \times 10^{-6}$ \\
\hline$B$ & 34641.699423 & $H_{K J}$ & $6.125 \times 10^{-6}$ \\
\hline$C$ & 29351.496787 & $H_{K}$ & $877.75 \times 10^{-6}$ \\
\hline$D_{J}$ & $56.00411 \times 10^{-3}$ & $h_{1}$ & $33.067 \times 10^{-9}$ \\
\hline$D_{J K}$ & $598.6775 \times 10^{-3}$ & $h_{2}$ & $32.462 \times 10^{-9}$ \\
\hline$D_{K}$ & 6.383483 & $h_{3}$ & $9.091 \times 10^{-9}$ \\
\hline$d_{1}$ & $-9.973179 \times 10^{-3}$ & $L_{K}$ & $-163.0 \times 10^{-9}$ \\
\hline$d_{2}$ & $-2.007905 \times 10^{-3}$ & $l_{1}$ & $-0.541 \times 10^{-12}$ \\
\hline$H_{J}$ & $0.01736 \times 10^{-6}$ & $l_{3}$ & $-0.472 \times 10^{-12}$ \\
\hline
\end{tabular}

Figure 1: Variation of partition function $Q$ versus kinetic temperature $T$. Solid line is for $Q_{1}$, obtained from equation (1) and the dotted line for $Q_{2}$, obtained from equation (2). 


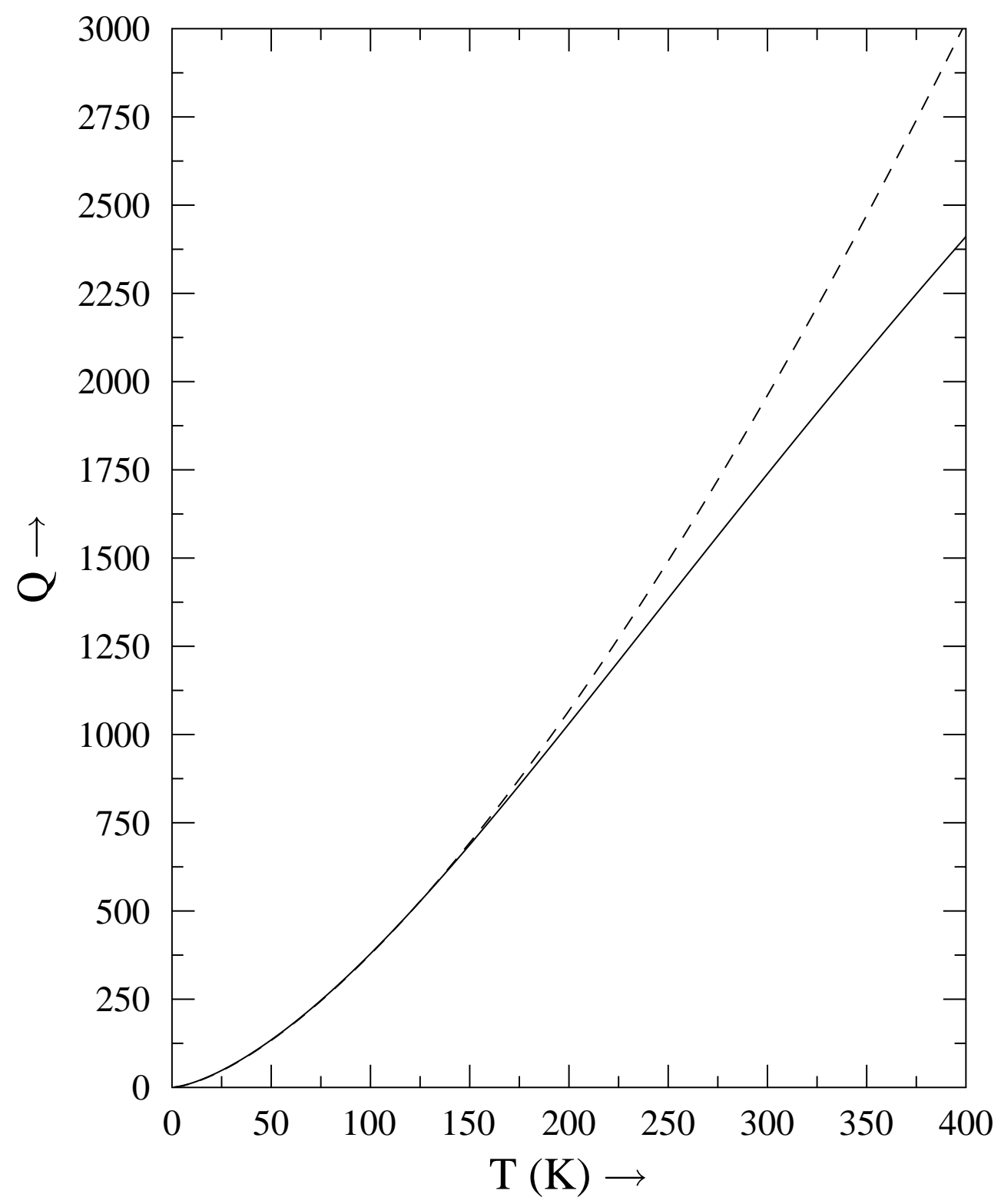

